\title{
A PLANNING BY USING DIGITAL TECHNOLOGY IN THE RECONSTRUCTION OF CULTURAL HERITAGE SITES - A CASE STUDY OF QIONG-LIN SETTLEMENT IN KINMEN AREA
}

\author{
W. B. Yang ${ }^{a, b}$ Y. N. Yen ${ }^{a *}$ \\ ${ }^{a}$ China University of Technology, 56 Sec. 3 ShingLong Rd., 116 Taipei, Taiwan - (wunbin, alexyen)@ cute.edu.tw \\ b National Taipei University of Technology, 1, Sec. 3, Zhongxiao E. Rd., 106 Taipei, Taiwan - t103859001@ntut.edu.tw
}

Commission II

KEY WORDS: Cultural Heritage, Preservation Planning, Planning Framework, GIS

\begin{abstract}
:
ICOMOS Florence Declaration in 2014, encourages an in-depth reflection on human values through cultural heritage and landscapes, which emphasizes the importance of historical heritage sites, in order to achieve the application of cultural heritage records through the public participation, sharing new technology platform and facilitation tools for knowledge diffusion, for instance. Nikos adopted digitized intangible cultural heritage within i-Treasures project to create a novel digital platform in 2016. Nowadays, the display platform developed based on geographic information system has been gradually accepted and widely used to distribute cultural heritage information, aiming to combine geography, time, events, issues, trends with the interactive maps to show the context of data changes from the consideration of planarity; for example, Burnaby City in Canada has cooperated with the Columbia University to create a navigation platform for guidance of tangible cultural heritage based on story maps in order to provide public recognition function.
\end{abstract}

In this study, Qiong-Lin Settlement in Kinmen Area was taken as an example to illustrate the developing process of an overall planning framework for reappearing the glory of historic settlements of cultural heritage sites with digital technology, which included tangible and intangible cultural heritage preservation and transmission planning, community participation and digital navigation programs. The digital technology with the GIS-based digital platform can provide more diverse and interesting information while using an intuitive, graphical user story mapping interface. So that tangible cultural heritage can be effectively understood, interpreted and preserved with the value-added methods, and also intangible cultural heritage can be continuously transmitted to establish a complete system of cultural heritage preservation. The main contents include several navigation technologies, such as 3D laser scanning, UAV images, photogrammetry, panorama, audio/video, geographic information systems etc.

\section{BACKGROUND}

\subsection{Florence Declaration}

In 2014, ICOMOS celebrated its 18th General Assembly and relevant heritage specialists dedicated to the theme of "The Florence Declaration", and proposed the following recommendations for actions:

1. Sharing and experiencing community identity through tourism and interpretation

2. Landscape as cultural habitat

3. Sustainability through traditional knowledge

4. Community-driven conservation and local empowerment

5. Emerging tools for conservation practice

This declaration reflects the aims of ICOMOS and its work with UNESCO in assessing tangible and intangible values associated with World Heritage properties, and the main aim of the 2014 Symposium was to protect the spirit of place and people's identity, as well as inclusion and participation of people and groups from a variety of cultures and to move forward in safeguarding and encouraging cultural diversity, so as to improve their quality of life. The preservation of cultural heritage has tended to consider the perspective of "locality", emphasizing the preservation model for "cultural" level. The localized culture is an integration of community experience and knowledge, in addition to the traditional norms and social values, it also has a significant correlation with the guiding, organizing, as well as standardizing people's way of life. Wherein, the community engagement will facilitate the local people to understand their own cultural values. The traditional activities and festivals conducted within the community can motivate people outside the community to interact with people inside the community. Also, through cultural heritage, dialogue can be created to link different cultures, which will help people in the field reviewing the historical culture at that time, to achieve a cultural awareness and participation.

Under the trend of technological progress, the method used for interpretation and demonstration of cultural heritage has changed, the original cultural heritage will be digitized, and even more, the old scenes will be reproduced, promoted and popularized through the Internet. It not only can achieve the goal and objectives for continue preservation and safeguarding of cultural properties, but also it can promote the education and knowledge associated with cultural properties.

2014 Florence Declaration encouraged an in-depth reflection on human values through cultural heritage and landscapes, which

* Corresponding author. This is useful to know for communication

with the appropriate person in cases with more than one author. 
emphasized the importance of historical heritage sites, in order to achieve the application of cultural heritage records through the public participation, sharing new technology platform and facilitation tools for knowledge diffusion, for instance, Nikos adopted digitized intangible cultural heritage within i-Treasures project to create a novel digital platform in 2016. Nowadays, the display platform developed based on geographic information system has been gradually accepted and widely used to distribute cultural heritage information, aiming to combine geography, time, events, issues, trends with the interactive maps to show the context of data changes from the consideration of planarity; for example, Burnaby City in Canada has cooperated with the Columbia University to create a navigation platform for guidance of tangible cultural heritage based on story maps in order to provide public recognition function.

\subsection{Chiung-Lin Settlement in the Kinmen County}

Currently, there are 150 places of traditional settlements in the Kinmen County of Taiwan, including conventional buildings, the western-style buildings and decorative arts, all of them have extremely rich humanities values. Due to the influence of military control period, it has still maintained a low degree of development for decades, but while facing the increased pressure on land development in recent years, the preservation of Kinmen's traditional architectures and settlements has reached a crisis point. Based on the special geographical location and historical background of Kinmen, the whole island has rich and diverse battlefields, the southern Fujian and overseas Chinese cultural properties that are in need of urgent protection. In view of this, the Bureau of Cultural Heritage, Ministry of Culture, selected the Kinmen region as a key area to be listed in the World Heritage in August 2009, and also launched a series of subsidies, it is hoping that the application for the inscription of world heritage with the World Heritage Committee will arouse public attention.

In the process of Kinmen's application for the World Heritage, it needs to review the preservation, safeguarding and redevelopment of Kinmen's culture, so that it is necessary to continuously integrate and implement all kinds of works, by means of the modification of regulations, repair of cultural properties, digital science and technology, safety and disaster prevention, public participation as well as the continuity and cultivation of culture.

It was found from the implementation of all kinds of works that "Cultural Field" cannot be preserved in the form of "single point", and instead, it should be preserved by considering the form of "dimension", while combining with the participation of local residents to implement the works related to preservation and redevelopment. The Chiung-Lin Settlement was officially registered in December, 2012 in accordance with the "Cultural Heritage Preservation Act", and during the period of 2013 2014, after combining with public participation and formation of a consensus, "The Preservation and Redevelopment Plan for the Chiung-Lin Settlement" was established accordingly. This plan combines with the project of historical representation will commonly promote the management and safeguarding of settlement preservation and activate overall development of the settlement.

The Chiung-Lin Settlement is the hot spot of existing 150 settlements in the Kinmen County, which still preserves more than 400 traditional historical buildings, and it is a legally registered settlement that has 8 national historic monuments, one county historic monument and three heritage buildings. In this historical site, it still preserves the annual festivals and important ordinary culture of the southern Fujian culture. Having abundant histories and diversified culture, the Chiung Lin Village completely preserves the southern Fujian culture, there are 8 ancestral shrines, the "Chiung-Lin ancestral worship ceremonies" held in the spring and autumn seasons annually has been registered as a cultural property, which is also considered as a previous and important heritage.

The title of this study is "To reappear the glory of thousand-year historic settlements of Chiung-Lin in the Kinmen area", and proposed a plan of "Reconstruction of Historical Scenes", and moreover, it uses various settlements as a platform, and adopts tangible cultural properties, such as national historic monuments and heritage buildings to be scenes, combining with the software of intangible cultural properties, such as ancestral worship, industries, folk customs, etc., and it is supported by digital technology and diversified plans in order to reappear the historical process of preservation and activation of conventional culture in a long period of time. This work is not only to reappear the historical sites, however, it is considering the historical sites as an important factor in the preservation of ordinary people's cultural activities and community engagement, and while supported by this project, it will be able to reappear the glory of tangible and intangible cultural properties of Chiung-Lin Settlement, i.e. the first historic settlements in the Kinmen area. It can be incorporated into ordinary people's living along with new cultural activities and science and technology resources in order to construe the foundation on the sustainable development of Chiung-Lin Settlement. This project will apply and extend practical experiences to some important works related to the preservation and redevelopment of other settlements in the Kinmen County. This project contains four sub-projects, including the preservation plan for tangible cultural properties, preservation plan for intangible cultural properties, community engagement and innovation activation plan, and technology navigation program for historical representation.

\section{THE PLANNING AND PERSPECTIVES OF RECONSTRUCTION OF HISTORICAL SITES AT THE CHIUNG LIN VILLAGE}

Chiung-Lin Settlement is the first settlement registered by legal authority, its cultural value is very high, the historical depth is very long, and both the tangible and intangible culture properties of the settlement are very rich, and because it is located in the center of Kinmen Island, so it had become a focus of the military defense during the confrontation between the two sides of the Strait. Under the influence of Chiung-Lin topography, the houses of settlement are divided into several groups with different orientations, in which, the building $\mathrm{s}$ in each group are facing the same direction, so there is no obvious characteristic of the northern and southern orientation, the settlement is generally constructed from the north and south into the comb-style layout, this comb-style layout is the same as the common layout used in the southern China, which can effectively reduce the radiation of sunlight, in addition to functions of winter-warm and summer-cool, and also it can set up a frontier pass to block the roadway, which is conducive to the establishment of defense system. The Chiung-Lin Settlement preserves a lot of traditional southern Fujian-style buildings, which are made of granite materials and developing a very unique traditional red brick culture. Such a unique the southern Fujian-style architecture was a product that was created under the influence of the Chinese and western cultural exchanges in the southern Fujian area since 17th century; the preservation system also included the traditional craftsman 
system and the construction technology, which was supported by the public and it is still a popular method used to build the houses in the present day. There are 332 the southern Fujianstyle traditional buildings located in the Chiung-Lin Settlement, which constitute the major spatial features of the Chiung-Lin Village.

The Chiung Lin Village was a single-surname village, i.e. Tsai, with one thousand years of history. The earliest member of clan that flight from Central Plain to the Kinmen and live in a community, which can be traced back to the later Tang Dynasty. During the Song Dynasty, ordinary people of Tsai clan began to organize themselves into a clan group and afterwards developed into a village in Kinmen, it is the central of everyday living that had a common ancestor of worship along with a common center of faith. The life structure was restricted by the traditional ethics, clan regulations managed the social order and formed an internal cohesion among the members of settlement; this situation had gradually developed until the Ming and Qing Dynasties, in which, the heritage of settlement with traditional intangible culture maintained until today, it was deemed to be a cultural treasure of the modern society. There are 11 tangible cultural properties in the Chiung-Lin Settlement, which include several national historic monuments, such as 7 places of Tsai Family Ancestral Shrines and one place of Yi Gu Tang, one county historic monument, i.e. Three Widows Chastity Arch, and two heritage buildings, i.e. Promoted Scholar Tsai's Residence (1) and (2); and intangible cultural properties include ancestral worship ceremony held in Tsai Family Ancestral Temple of Chiung-Lin Settlement and Wind-lion Gods.

\subsection{Description of objectives}

1. To strengthen the investigation, research and planning of the cross-domain integration of cultural properties in the Chiung-Lin Settlement.

2. To fulfill local public participation in the Chiung-Lin Settlement, and promote multiculturalism.

3. To establish the integrity of basic data and digital archives associated with cultural properties at the Chiung-Lin Settlement.

4. To preserve intangible cultural properties as well as to guide and cultivate folk activities.

5. Developing the preservation of digitization of cultural property, and promoting the establishment and navigation of science and technology resources for historical spaces.

6. To share the experience of reconstruction of historical sites, and push forward preservation and activation of settlements in the Kinmen County.

\subsection{Perspectives for execution}

Under the increasing pressure of globalization for continuous development in the 21 st century, the definition of preservation of cultural properties is not only a simple static building preservation; to verify the value of cultural properties along with the consideration of authenticity and wholeness. It needs to consider the relationship and positioning between tangible and intangible cultural properties, for implementing sustainable development and preservation of cultural properties has become an international general rule. Under the influence of globalization, using digital technology to assist the preservation of cultural properties has become a major trend, and hence, how to record, integrate and add values to tangible and intangible cultural properties through science and technology is deemed to be one of the most urgent issues in the present day.
Affected by international trends, the recognition of cultural properties has tended to be diversification, and the interpretation and presentation of values is not merely depending on the architecture, history, and therefore, there is a necessity for integrating multidisciplinary and transdepartmental efforts, and in order to effectively demonstrate the values of cultural properties, it must be changed from a single point of architecture to the "field" with lines and dimensions, so as to effectively demonstrate the significance and value provided by cultural properties to the country, domestic area and the public. Based on the diversification of values of cultural properties, along with the trend of changing the concept of field from single point to dimensions as well as the operation of digital technology, the project of "Reconstruction of Historical Scenes" will facilitate in linking the relationship between land, people and cultural properties in order that tangible cultural properties will be effectively understood, interpreted, preserved and value-added, allowing intangible cultural properties be able to sustainable inheritance, under this circumstance, it will make cultural properties be able to combine with other industries to construct a complete preservation system for cultural properties. The relationships involve the execution of this project is as follows:

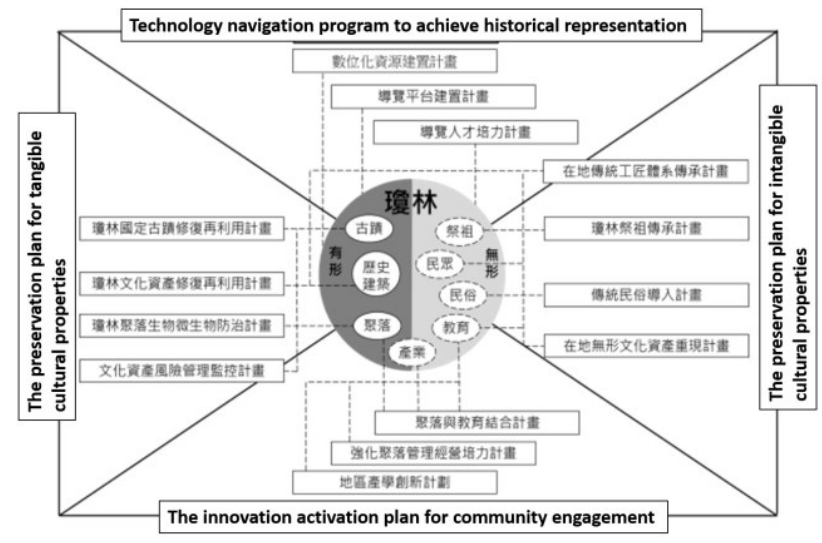

Figure 1. The relationship diagram for execution of this project

\section{THE PLANNING FOR PROJECT CONTENTS ASSOCIATED WITH RECONSTRUCTION OF HISTORICAL SITES AT THE CHIUNG-LIN VILLAGE}

This project primarily contains four sub-projects, including the preservation plan for tangible cultural properties, preservation plan for intangible cultural properties, community engagement and innovation activation plan, and technology navigation program for historical representation, the major architecture is as follows:

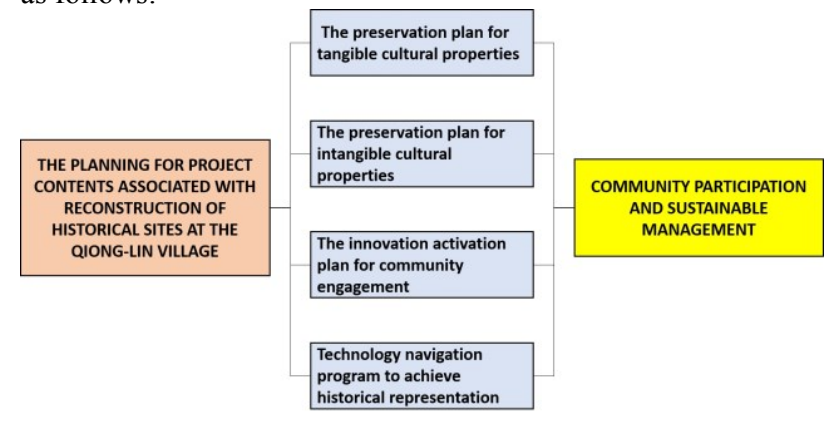

Figure 2. The architecture diagram of this project 


\subsection{Sub-project A - The preservation plan for tangible cultural properties}

The relevant tangible cultural properties located in the ChiungLin Settlement, which are required to be preserved, include the national historic monument - Tsai Family Ancestral Shrine $(8$ Places), Chiung-Lin underground tunnel, Wind-lion Gods, Door-god painting of Tsai Family Ancestral Temple and other important tangible cultural properties. The primary implementation process are to verify the historical origin of cultural properties, analyzes and determine the original appearance of the structures and crafting method of artisans, investigate and analyze the damage conditions and safety of its structures, draw up repair methods etc., which aims to recover and preserve original appearance of tangible cultural properties by means of investigation and analysis as well as abovementioned repair methods.

In addition to the self-damage, the tangible cultural properties in the Chiung-Lin Settlement are also harmed by both biological and microbiological activities, and therefore, the biological and microbiological control plans are put forward, which shall be combined with the definition of biology and microorganism, as well as the establishment of routine management and safeguarding methods, in order to achieve the goal of effective prevention of the tangible cultural properties in the Chiung-Lin Settlement being harmed by the biological and microbiological activities.

Except above-mentioned Tsai Family Ancestral Shrine and Door-god of Tsai Family Ancestral Temple, traditional shophouses on Qiongyi Road are deemed to be important historical places required for the preservation and safeguarding. Qiongyi Road is the main north-south road that runs through the Chiung-Lin Settlement. It was openly up within the period of Battlefield Government Affairs, there are many shop-houses constructed on both sides of the road to meet the requirements of military and civilian consumption. It used to be a very important historical scene in central Kinmen area. Alter official withdrawal of R.O.C. troops from the Kinmen County, some shop-houses have been reconstructed but quite a lot of them have been idle or become empty houses. Through procedure of survey and repair, it will be able to reproduce the elegance of that time again, and also it can be considered as the specific space fields in this project for bring in various industries (such as, restaurants, experience, cultural and creative Industries, etc.).

\subsection{Sub-project B - The preservation plan for intangible cultural properties}

The Chiung Lin Village has abundant intangible cultural properties, including heritage of ancestral worship practiced at the Chiung Lin Village and local traditional folklore. The ancestral worship practiced at the Chiung Lin Village is recognized as a legal cultural property, which is also deemed to be an important cultural target in Chinese Society. While facing several problems such as insufficient resources and faults, which shall be settled by strengthening various hardware and software, text construction, planning and implementation of activities, transmission, cultivation and experience, promotion and execution of strategies, and integration of cultural marketing. The above-mentioned coping method includes the enhancement of basic hardware and software, and in addition, the Chua Family's ancestral worship ceremony will be constructed through local historical literature and contemporary dictation associated with the issues of Chiung Lin Village. With the assistance from local community organizations, systematically planning activities and reproducing the unique historical scenes, it can not only pass to the next generation, but also it will allow visitors to profoundly experience the local culture to fulfill the promotion and marketing for intangible culture of Chiung Lin Village, and meanwhile achieving the goals and objectives for the sustainable development of community by driving the development of related industries.

Except above-mentioned intangible cultural properties, the inheritance of local traditional crafting system has been considered as an important intangible cultural property at the Chiung Lin Village. The method of implementation is to combine the traditional building maintenance autonomy regulations existing in the Kinmen County and craftsmen certification mechanism, to employ the registered and designated preservation specialists for cultural properties to be the major resource of teachers, who will implement the traditional craftsman succession plan at the repair and reuse sites of cultural properties.

\subsection{Sub-project $C$ - The innovation activation plan for community engagement}

While combining the preservation of tangible and intangible cultural properties, and to guide the new Industries with diversified innovation management in the historical scenes, the guidance programs include home stay facilities, special gourmets, tourism experience, the research, development and production of cultural and creative products, etc., which will not only create many employment opportunities and but also it will encourage the return of young people, and meanwhile, timely introducing the latest digital technology will definitely increase their output values.

In order to make the intangible cultural properties of the settlement can be deeply rooted into local education, it is proposed to strengthen the "Traditional Gran Cassa Team of Kinmen Kaishiuan Primary School" (the most massive team in the Kinmen County), and in addition, to combine it with cultural properties, native education and deepen the education of cultural properties. While cooperating with local NGO in the Kinmen County, this project will carry out the preservation of historical fields by means of active participation of local residents, including operation, management, disaster prevention and other training programs, as well as the establishment of household defense mechanism.

\subsection{Sub-project D - Technology navigation program to achieve historical representation}

By means of digitization technology (audio \& video, multimedia), hence, a complete documentation related to various tangible and invisible cultural resources in the Chiung Lin Village can be established and used as an important resource for historical sites, and in which, laser scanning measurement and mapping data contains 360-degree digital panoramic images, which can be used to are made 10 sets of 3D scene models for virtual reality (VR) applications, in order to provide the functions of digital navigation and demonstrate, and furthermore, this project also produced 8 digital films and organized microfilm workshops and competitions.

For promoting the use of digitized resources, it needs to assist the communities to spontaneously established common multilingual navigation platforms and integrated various results and digital resources from this project, including Internet, digital auto-guiding system in addition to the training of local navigation talents and so on. To integrate tangible and intangible historical sites with dynamic activities in order to provide value-added applications on both the tangible and 
intangible cultural properties, and moreover, it is necessary to develop a series of game-based learning materials and conduct marketing and promotion for relevant teaching materials as well.

\section{THE INTEGRATED PRESENTATION OF DIGITIZED RECONSTRUCTION OF HISTORICAL SITES}

The integrated digital contents of reconstruction of historical sites at the Chiung Lin Village provided by this study was shown in Fig. 3, in which, the relevant contents would be firstly integrated by using Geographic Information System, and then be demonstrated with theme stories.

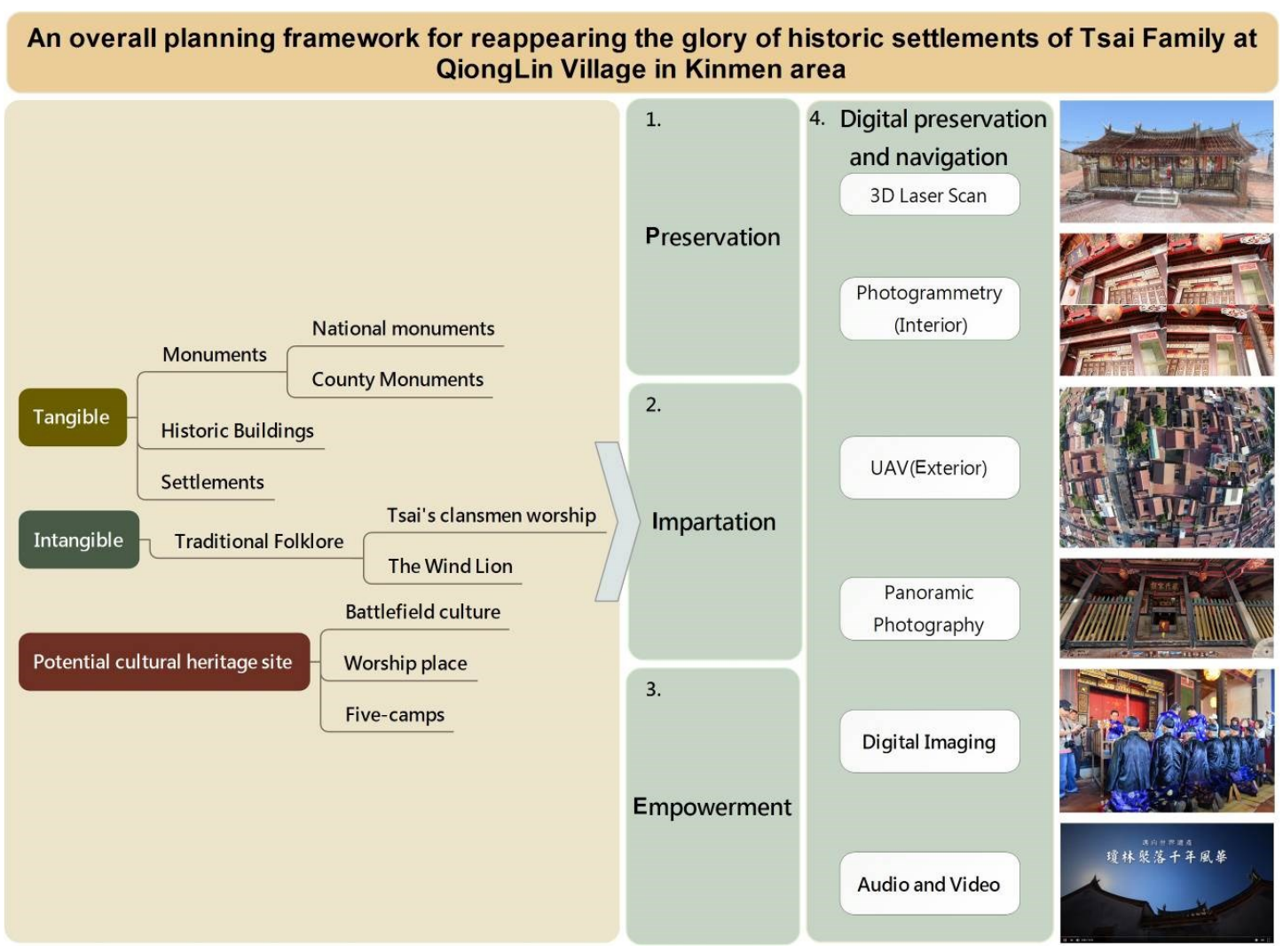

Figure 3. Digital contents associated with reconstruction of historical sites at the Chiung Lin Village

The Geographic Information System can process, summarize, classify and calculate a great deal of geographic information, whose meaning can be carefully Interpreted by individual user, and thus, affecting such user's behaviors and decision making. Additionally, it can express natural and humanistic phenomena, and maps have the characteristics of intuition, visibility and measurability. Therefore, positioning and quantitative data will be obtained through procedures of map measurement.

Derived from the Geographic Information System, which was focusing only on the spatial representation in the past, Story Map has added the concept of time, and uses the storytelling technique to express relevant documentaries. The archival information that existed about a given historical event or time in the past, currently, it can effectively convey the changes of overall time and space background while combining with the space information provided by the Geographic Information System, the results of this project are shown in Fig. 4.

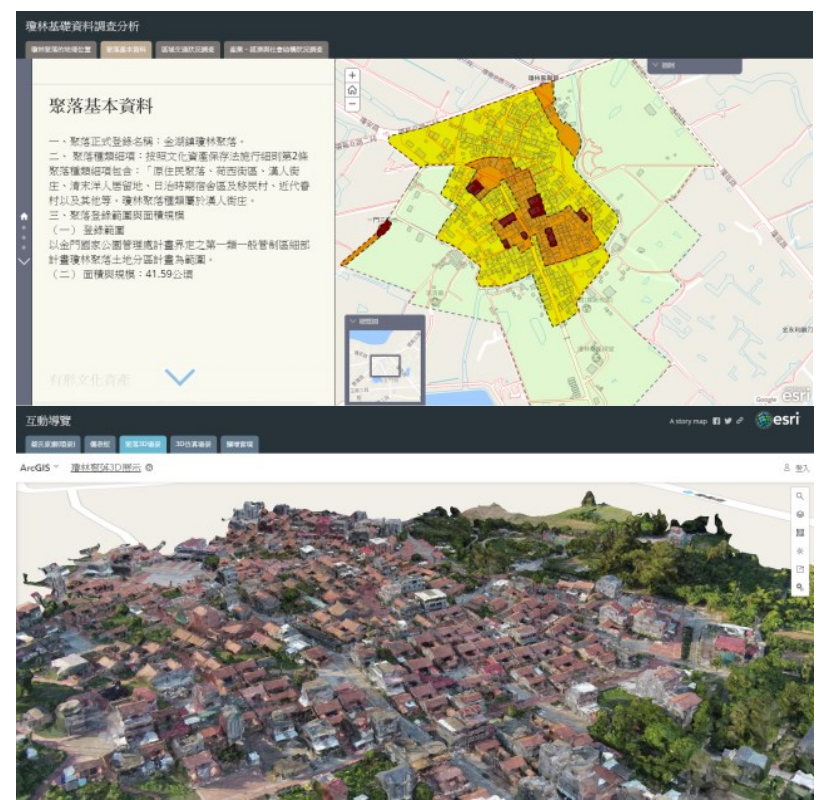

Figure 4. A result of integrated presentation of digitized reconstruction of historical sites at the Chiung Lin Village 


\section{CONCLUSIONS}

Following with technological progress, the integration of digitized presentation technology in terms of digital preservation of cultural heritage has become a necessary trend. Except the digital preservation of tangible cultural heritage, the preservation of intangible cultural properties has gradually been changed from traditional means into digital preservation, applications and developments. The transfer of intangible cultural properties is not easy, however, it will increase the value of intangible cultural properties and facilitate the promotion and demonstration of intangible cultural properties with the method of digitization, and in addition, while integrating with the Geographic Information System, story maps will then be able to provide more diversified and interesting information presentation and inheritance through an intuitive, graphical operation Interface, instead of original web pages that only contain texts and pictures on it.

\section{REFERENCES}

Grammalidis, N., \& Dimitropoulos, K. (2015). Intangible Treasures-Capturing the Intangible Cultural Heritage and Learning the Rare Know-How of Living Human Treasures.

Heritage Burnaby, https://heritageburnaby.ca/

ICOMOS, 2014. Florence declaration.

Wellington City Council, http://wellington.govt.nz/ 